\title{
Isolation and Characterization of Larvicidal Phenolic Acids from Kotschya thymodora
} Leaves

\author{
*1,2DANIEL, IJ; ${ }^{2}$ INNOCENT, E; ${ }^{1}$ SEMPOMBE, J; ${ }^{1}$ MUGOYELA, V; ${ }^{3}$ FOSSEN, T \\ ${ }^{*}$ Department of Medicinal Chemistry, School of Pharmacy, Muhimbili University of Health and Allies Sciences, P. O. Box 65013, Dar es \\ salaam, Tanzania. \\ ${ }^{2}$ Department of Biological and Pre-clinical Studies, Institute of Traditional Medicine, Muhimbili University of Health and Allies Sciences, \\ P. O. Box 65001, Dar es salaam, Tanzania. \\ ${ }^{3}$ Department of Chemistry and Centre for Pharmacy, University of Bergen, Allégt. 41, N-5007 Bergen, Norway. \\ *Corresponding Author Email: innocentdaniel42@gmail.com
}

\begin{abstract}
Malaria is a vector borne disease responsible for high morbidity, mortality and poverty in many tropical and subtropical countries. The disease is transmitted through a bite from an infected female Anopheles mosquito, amongst which Anopheles gambiae s.s serves as the most prevalent vector. The control of An. gambiae s.s population can therefore lead to a reduction in malaria spreading. Previous studies have reported the crude extracts of Kotschya thymodora to be active against the larvae of An. gambiae s.s and Culex quinquefasciatus. In this report the phytochemical study on the crude aqueous ethanolic extract of $K$. thymodora leaves led to isolation of vanillic acid (1) and protocatechuic acid (2). The structures of these compounds and mosquitoes larvicidal activity against An. gambiae s.s were established by using spectroscopic techniques and WHO protocol of 1996 respectively. The two phenolic acids exhibited a moderate mosquito larvicidal activity with $\mathrm{LC}_{50}$ of $77.35 \mu \mathrm{g} / \mathrm{mL}$ (vanillic acid, 1) and $62.4 \mu \mathrm{g} / \mathrm{mL}$ (protocatechuic acid, 2) after $48 \mathrm{hrs}$ exposure time. This is the first report on the isolation of the two phenolic acids from plants belonging to the genus Kotschya and their larvicidal potential against An. gambiae s.s.
\end{abstract}

DOI: https://dx.doi.org/10.4314/jasem.v24i8.26

Copyright: Copyright (C) 2020 Daniel et al. This is an open access article distributed under the Creative Commons Attribution License (CCL), which permits unrestricted use, distribution, and reproduction in any medium, provided the original work is properly cited.

Dates: Received: 07 June 2020; Revised: 27 July 2020; Accepted: 26 August 2020

Keywords; larvicidal activity, vanillic acid, protocatechuic acid, Anopheles gambiae s.s and Kotschya thymodora

Anopheles mosquitoes are the main vectors responsible for transmission of malaria. In the life cycle of malaria parasites, these mosquitoes serve as definitive host permitting their sexual multiplication and maturation to infective forms (Sporozoite) (Cox, 2010). Anopheles gambiae s.s, is known to be the most prevalent vector of malaria parasites and also a key transmitter of Plasmodium falciparum, a protozoan that causes severe forms of malaria (Leong et al., 2003). The parasite $P$. falciparum is also the main cause of deaths due to malaria in endemic countries located in Africa, South-east Asia and Western Pacific region (WHO, 2019). About $94 \%$ of the malaria deaths worldwide occur in sub-Saharan Africa where the climatic conditions favor the survival and propagation of An. gambiae s.s (Leong et al., 2003). Many of the countries located in malaria endemic regions are also characterized by economic underdevelopment. This can be partly contributed by high malaria burden which imparts a significant economic loss to households and national at large. The economic loss due to malaria is usually the result of direct and indirect costs incurred for its prevention, diagnosis and treatment (Teklehaimanot and Mejia, 2008). For long time the use of synthetic insecticides and antimalarial drugs have served as the mainstay methods for malaria control. Insecticides are normally used with the aim of eliminating An. gambiae s.s vector whereas antimalarial drugs for inhibiting the passage of Plasmodium gametocytes from infected to uninfected individual (Kimbi, 2012). The main synthetic insecticides used in the mosquito vector control includes those derived from pyrethroid, carbonates, organochlorines and organophosphates. However, some current reports show that some mosquitoes have developed resistance against these synthetic insecticides and therefore their efficiency in controlling malaria transmission is reduced (Yewhalaw et al., 2011). On the other hand, the malaria parasites have also shown to develop resistance towards many commonly used antimalarial drugs, hence posing a threat for its continued transmission (Kokwaro, 2009). These two factors present the major challenges towards the eradication of malaria and its vectors (WHO, 2019). In order to improve the control of malaria transmission, it is therefore important to overcome the resistance of malaria parasites and mosquito vectors. This calls for 
an effective malaria vector control method from other alternative sources. Plants offer a wide range of chemical substances that have insecticidal properties. For instance, the ethanolic crude extracts of Kotschya thymodora (Baker F) leaves and roots have previously been reported to be potent larvicides against $A n$. gambiae s.s (Daniel et al., 2020) and $C x$. quinquefasciatus (Innocent et al., 2012). The leaves extract exhibited larvicidal activity against $A n$. gambiae s.s with $\mathrm{LC}_{50}$ of $16.35 \mu \mathrm{g} / \mathrm{mL}$. The mean mortality recorded for the crude ethanolic leaves extract of $K$. thymodora at $50 \mu \mathrm{g} / \mathrm{mL}$ was $\geq 80 \%$ after $48 \mathrm{hrs}$ exposure. Another phytochemical study on crude ethanolic leaves extract of $K$. thymodora afforded the isolation of cycloartenone. The compound exhibited a mild larvicidal activity against An. gambiae s.s with a mean of mortality of $40 \%$ at 50 $\mu \mathrm{g} / \mathrm{mL}$ after $72 \mathrm{hrs}$ exposure (Innocent et al., 2015). The higher larvicidal potential reported for the crude extracts of $K$. thymodora compared to that of cycloartenone makes it interesting to carry out further isolation of other active compound(s). Therefore, this study reports the structural characterization of two phenolic acids from $K$. thymodora leaves extract and their larvicidal activity against An. gambiae s.s.

\section{MATERIALS AND METHODS}

Collection and identification of plant materials: The leaves of K. thymodora were collected from Njombe region (GPS coordinates 9056' 11.2 E 34034' 52.6) with voucher specimen number FMM 3628. The collection and authentication were done by Mr. Haji Seleman, a Botanist from the Department of Botany at the University of Dar es salaam. The specimen is deposited in the Herbarium of Institute of Traditional Medicine (ITM) at Muhimbili University of Health and Allied Sciences (MUHAS).

Preparation of plant materials and extraction procedure: The plant materials were chopped into small pieces, dried at room temperature $\left(28 \pm 2^{\circ} \mathrm{C}\right)$ and then milled into course powder. Extraction of powdered plant materials was done in $20 \%$ aqueous ethanol for $72 \mathrm{hrs}$ with occasional shaking. The extracts were filtered, concentrated using rotary evaporator at $25^{\circ} \mathrm{C}$ and then stored in the refrigerator.

Chromatographic isolation of compounds (1) and (2): About $75 \mathrm{~g}$ of the dried aqueous ethanolic extract from $K$. thymodora leaves was fractionated through vacuum liquid chromatography (VLC) using silica gel 60 (230$400 \mathrm{mesh})$ to give fractions from petroleum ether, ethyl acetate, and methanol mobile phases. The ethyl acetate fraction was concentrated on a rotary evaporator at $25{ }^{\circ} \mathrm{C}$ and subjected to further fractionation on amberlite XAD-7 column. Five fractions were collected from the following elutions: Super distilled water (fractions 1), 20\% methanol/water (fractions 2), 50\% methanol/water (fractions 3), 80\% methanol/water (fractions 4 ), and $100 \%$ methanol (fractions 5). Fraction 2 was concentrated using a rotary evaporator and subjected to Sephadex LH-20 column chromatography. A total of $88 \mathrm{sub}$-fractions were collected using the following mobile phases: methanol/water/TFA at 30:70:0.2 (sub-fractions 2.1 - 2.18), methanol/water/TFA at 60:40:0.2 (sub-fractions 2.19 - 2.25), and methanol/water/TFA at 80:20:0.2 (sub-fraction 2.26 2.88). All sub-fractions were subjected to analysis by HPLC and each of fractions 2.42 and 2.48 contained one major compound. These fractions were concentrated on a rotary evaporator at $25^{\circ} \mathrm{C}$, and thereafter dried under nitrogen gas to give a brownish amorphous powder named as compound $\mathbf{1}(38 \mathrm{mg})$ and compound $2(46 \mathrm{mg})$ respectively.

Qualitative UV detection of compound 1 and 2 using Analytical HPLC-DAD: The UV absorption and retention time for vanillic acid (1) and protocatechuic acid (2) were recorded online using an Agilent technologies 1260 HPLC instrument coupled to a Diode Array Detector $(190-600 \mathrm{~nm})$. The analyses were performed on a reversed phase column (C-18). The HPLC pump system was first purged with solutions A (Super distilled water with $0.2 \% \mathrm{~V} / \mathrm{V}$, trifluoracetic acid), B (acetonitrile with $0.2 \% \mathrm{~V} / \mathrm{V}$, trifluoracetic acid), $\mathrm{C}(70 \%$ methanol/water) and $\mathrm{D}$ ( $70 \%$ acetonitrile/water) at $3 \mathrm{~mL} / \mathrm{min}$ for 5 minutes. A $15 \mu \mathrm{L}$ volume of each sample was injected by an auto injector. The flow rate was set at $1 \mathrm{~mL} / \mathrm{min}$. Analysis was performed using gradient elution from $90 \% \mathrm{~A} / \mathrm{B}$ to $10 \% \mathrm{~A} / \mathrm{B}$ within 55 minutes. The major compounds in the fractions 2.42 and 2.48 had retention times of $7.42 \mathrm{~min}$ and $6.80 \mathrm{~min}$ respectively. The compound in fraction 2.42 displayed UV absorption maxima at 262 $\mathrm{nm}$ and $292 \mathrm{~nm}$. The compound in fraction 2.48 (protocatechuic acid) displayed UV absorption maxima at $\lambda_{\max } 260 \mathrm{~nm}$ and $294 \mathrm{~nm}$.

NMR spectroscopic analyses: The structures of the isolated compounds were determined using $1 \mathrm{D}$ and $2 \mathrm{D}$ NMR spectroscopic data recorded on a Bruker 850 $\mathrm{MHz}$ instrument. The NMR experiments performed were ${ }^{1} \mathrm{H}-\mathrm{NMR},{ }^{13} \mathrm{C}-\mathrm{NMR},{ }^{1} \mathrm{H}-{ }^{1} \mathrm{H} \quad \mathrm{COSY},{ }^{1} \mathrm{H}-{ }^{13} \mathrm{C}$ Edited HSQC and ${ }^{1} \mathrm{H}_{-}{ }^{13} \mathrm{C}$ HMBC. The spectrometer frequencies (SF) was set at $850.13 \mathrm{MHz}$ and 213.77 $\mathrm{MHz}$ for ${ }^{1} \mathrm{H}$ and ${ }^{13} \mathrm{C}$ respectively. The samples were dissolved in deuterated DMSO and the experimental temperature was maintained at $298 \mathrm{~K}$.

Mosquito Larvae rearing: Larvae of An. gambiae s.s for bioassay of the isolated compounds were collected 
from the insectary at the Institute of Traditional Medicine (ITM), Muhimbili University of Health and Allied Sciences (MUHAS) which rears a colony obtained from National Institute for Medical Research (NIMR), Amani Research Centre in Tanga region of Tanzania. The mosquito larvae were reared in distilled water. Instars stage 1 and stage 2-4 were fed on yeast and fish food (Tetramin $®)$ respectively.

Larvicidal bioactivity: The larvicidal bioactivity was determined following the WHO protocol of 1996 with minor modification. Stock solutions were prepared by dissolving $5 \mathrm{mg}$ of the pure compound in $1 \mathrm{~mL}$ of DMSO. From this, $50 \mu \mathrm{g} / \mathrm{mL}, 25 \mu \mathrm{g} / \mathrm{mL}, 12.5 \mu \mathrm{g} / \mathrm{mL}$, and $6.25 \mu \mathrm{g} / \mathrm{mL}$ solutions were prepared in $100 \mathrm{~mL}$ beakers for testing. For all the assays 15 healthy third instars larvae were placed in $50 \mathrm{~mL}$ of the appropriate concentration in distilled water. Four replicates of each concentration were prepared and the tests were carried out three times. The control experiment was set up in dimethyl sulfoxide (DMSO) at $1 \% \mathrm{~V} / \mathrm{V}$ in distilled water. The cumulative mortality data were recorded at 24 and $48 \mathrm{hrs}$ post exposure of the mosquito larvae to treatments. In the entire period of larval rearing and larvicidal activity testing the room temperature and relative humidity were maintained at $28 \pm 2^{\circ} \mathrm{C}$ and $75 \pm 10 \%$ respectively.

Statistical analysis: The cumulative percentage mortalities and standard deviations were calculated using Microsoft Excel (2013). Probit analysis was used to estimate the $\mathrm{LC}_{50}$ and $\mathrm{LC}_{90}$ values and their standard deviations from the regression analysis done by using Graphpad prism (version 5.0). Statistical significance testing of the estimates was determined using analysis of variance (ANOVA) followed by Tukey multiple comparison test.

\section{RESULTS AND DISCUSSION}

Characterization of phenolic acids by NMR spectroscopy: The ${ }^{13} \mathrm{C}$ NMR spectrum of compound $\mathbf{1}$ exhibited signals for aliphatic and aromatic carbons. The aliphatic signal appears at $\delta 55.61$ while the most deshielded carbon appears at $\delta 167.28$. The aromatic region of ${ }^{1} \mathrm{H}$ NMR spectrum of compound 1 showed three protons at $\delta 7.43, \delta 7.42$ and $\delta 6.82$. This was further confirmed by COSY spectrum which showed homonuclear couplings of the neighboring proton at $\delta$ $7.43(2.0 \mathrm{~Hz} ; \mathrm{H}-2), \delta 7.42(8.1 \mathrm{~Hz}, 2.0 \mathrm{~Hz} ; \mathrm{H}-6)$ and $\delta$ $6.82(8.1 \mathrm{~Hz} ; \mathrm{H}-5)$ characteristic of ABX system. The HSQC was used to assign the protons to their respective carbons. Furthermore, the aliphatic region of the ${ }^{1} \mathrm{H}$ NMR spectrum of compound 1 showed the presence of $3 \mathrm{H}$ as singlet at $\delta 3.79$ which is in accordance with a methoxy group. The HMBC experiment showed correlations between protons of the methoxy group $\left(-\mathrm{OCH}_{3}\right)$ at $\delta 3.79$ and $\delta 147.30(\mathrm{C}$ 3 ). The substituent at position 1 of the aromatic ring was identified as carboxylic acid due to carbonyl carbon observed at $\delta 167.28$ in the $1 \mathrm{D}{ }^{13} \mathrm{C} \mathrm{NMR}$ spectrum and the carboxylic hydroxyl proton at $\delta$ 12.70 observed in the 1D ${ }^{1} \mathrm{H}$ NMR spectrum. The substituent at 4-position of the aromatic ring was identified as a hydroxyl group by the phenolic proton observed resonating at $\delta 9.82$ in the $1 \mathrm{D}{ }^{1} \mathrm{H}$ NMR spectrum. Thus, compound $\mathbf{1}$ was identified as vanillic acid. The NMR spectra of compound $\mathbf{2}$ showed great similarity with compound $\mathbf{1}$ but having few exceptions. The $1 \mathrm{D}{ }^{1} \mathrm{H}$ NMR spectrum of compound $\mathbf{2}$ showed a similar ABX resonance and coupling system as in compound 1 except for additional broad singlet proton signal at $\delta 9.28$ which is in accordance with a hydroxyl group of the aromatic ring and was determined to be at C-3 according to $\mathrm{HMBC}$ correlations (Figure 1). Thus, compound $\mathbf{2}$ was identified as protocatechuic acid. The spectral data for vanillic acid (1) and protocatechuic acid (2) are similar to those published by (Ghareib et al., 2010) and (An et al., 2006) respectively. Complete assignments of protons and carbon shifts of the two phenolic acids are shown in Table 1 and Figure 1.

Bioactivity of the isolated compounds: The results of larvicidal test indicate that both phenolic acids possess a moderate larvicidal activity against An. gambiae s.s. The $\mathrm{LC}_{50}$ and $\mathrm{LC}_{90}$ of the phenolic acids estimated from the mean cumulative mortalities recorded after $48 \mathrm{hrs}$ are shown on Table 2 . Statistical analysis at $\mathrm{P} \leq$ 0.05 reveals significant difference between the $\mathrm{LC}_{50}$ and $\mathrm{LC}_{90}$ values of the individual compound, but not between the two compounds. No mortalities were recorded in the control experiments. The control of $A n$. gambiae s.s mosquito by using larvicides offers a great advantage not only to the reduction or elimination of malaria transmission but also avoids the nuisance bites of their adults (Ghosh et al., 2012). Studies have shown that plants can serve as source of insecticidal compounds which include some of commercially available products such as pyrethrin, azadirachtin, rotenone, nicotine and capsaicin (Pavela, 2016).

The crude ethanolic extracts of some plants belonging to genus Kotschya have shown to exhibit mosquito larvicidal properties against various mosquito species. Previous phytochemical studies have demonstrated the larvicidal potential of some compounds isolated from the polar extracts of members from this genus. For instance, a recent study has reported the isolation of ent-halim-1(10)-ene-15oic acid and 3-O-methyl-D-chiro-inositol from the ethanolic extracts of Kotschya uguenensis leaves. 
Table 1. ${ }^{1} \mathrm{H}$ and ${ }^{13} \mathrm{C}$ chemical shift values (ppm) and coupling constants (Hz) of vanillic acid (1) and protocatechuic acid (2) isolated from K. thymodora leaves. Solvent DMSO-D 6 at 298K.

\begin{tabular}{|c|c|c|c|c|c|c|c|c|}
\hline \multirow{2}{*}{$\begin{array}{l}\text { Carbon } \\
\text { number }\end{array}$} & \multicolumn{2}{|l|}{ Vanillic acid (1) } & \multirow{2}{*}{$\stackrel{\mathrm{HMBC}}{\longrightarrow} \mathrm{C})$} & \multirow{2}{*}{$\overbrace{(\mathrm{H} /)}^{\mathrm{COSY}}$} & \multicolumn{2}{|c|}{ Protocatechuic acid (2) } & \multirow{2}{*}{$\begin{array}{c}\mathrm{HMBC} \\
(\mathrm{H} \longrightarrow \mathrm{C})\end{array}$} & \multirow{2}{*}{$(\mathrm{H} / \mathrm{H})$} \\
\hline & $\delta^{1} \mathrm{H}$ & $\delta^{13} \mathrm{C}$ & & & $\delta{ }^{1} \mathrm{H}$ & $\delta{ }^{13} \mathrm{C}$ & & \\
\hline 1 & & 121.70 & & & & 122.32 & & \\
\hline 2 & $7.43 \mathrm{~d} 2.0$ & 112.79 & $\mathrm{C}-1, \mathrm{C}-3$ & H-6 & $7.33 \mathrm{~d} 2.1$ & 116.99 & $\mathrm{C}-1, \mathrm{C}-3$ & $\mathrm{H}-6$ \\
\hline 3 & & 147.30 & & & & 145.34 & & \\
\hline 4 & & 151.18 & & & & 150.46 & & \\
\hline 5 & $6.82 \mathrm{~d} 8.1$ & 115.09 & C-4 & H-6 & $6.78 \mathrm{~d} 8.6$ & 115.59 & $\mathrm{C}-4$ & H-6 \\
\hline 6 & $7.42 \mathrm{dd} 8.1,2.0$ & 123.54 & $\mathrm{C}-1, \mathrm{C}-5$ & $\mathrm{H}-2, \mathrm{H}-5$ & $\begin{array}{l}7.28 \mathrm{dd} \\
8.6,2.1\end{array}$ & 122.09 & C-1, C-5 & H-2, H-5 \\
\hline 7 & & 167.28 & & & & 167.74 & & \\
\hline $3-\mathrm{OH}$ & & & & & $9.28 \mathrm{~s}, \mathrm{br}$ & & C-3 & \\
\hline $3-\mathrm{OCH}_{3}$ & $3.79 \mathrm{~s}$ & 55.61 & C-3 & & & & & \\
\hline $4-\mathrm{OH}$ & $9.82 \mathrm{~s}$ & & $\mathrm{C}-3$ & & $9.67 \mathrm{~s}, \mathrm{br}$ & & C-3 & \\
\hline $1-\mathrm{COOH}$ & $12.70 \mathrm{~s}$ & & & & $12.29 \mathrm{~s}, \mathrm{br}$ & & & \\
\hline
\end{tabular}

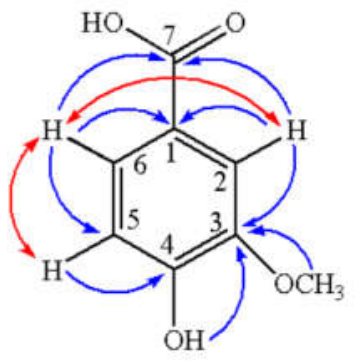

1

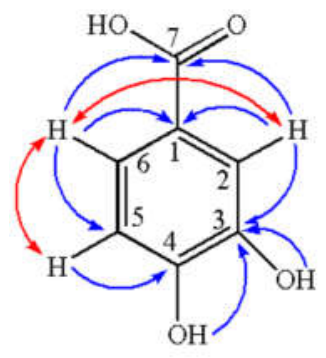

2

Fig 1: COSY and $\longrightarrow$ HMBC important correlations for compounds 1 and 2.

Table 2: $\mathrm{LC}_{50}$ and $\mathrm{LC}_{90}$ values for vanillic acid (1) and protocatechuic acid (2) estimated from mean mortalities of the mosquito larvae

\begin{tabular}{llllc}
\multicolumn{5}{c}{ recorded after $48 \mathrm{hrs}$ exposure time. } \\
\hline Compound & $\begin{array}{l}\mathrm{LC} 50 \\
(\mathrm{LCL}-\mathrm{UCL})\end{array}$ & $\begin{array}{l}\mathrm{LC} / \mathrm{mL}) \\
(\mathrm{LCL}-\mathrm{UCL})\end{array}$ & $\begin{array}{l}\text { Regression } \\
\text { equation }\end{array}$ & $\mathrm{R} 2$ \\
\hline Vanillic acid (1) & $62.43(53.24-71.60)$ & $190.49(171.73-209.25)$ & $\mathrm{Y}=2.73 \mathrm{X}+0.01$ & 0.96 \\
Protocatechuic acid (2) & $77.41(65.79-89.14)$ & $264.46(205.66-323.26)$ & $\mathrm{Y}=2.40 \mathrm{X}+0.46$ & 0.86 \\
\hline
\end{tabular}

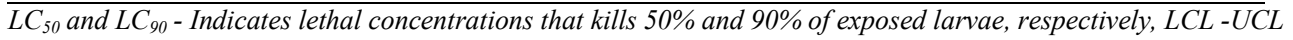
indicate Lower-class limit and Upper-class limit at $95 \%$ confidence level respectively. Number of replicates is three

The two compounds possess activity against $A n$. gambiae s.s with $\mathrm{LC}_{50}$ values of $30.05 \mu \mathrm{g} / \mathrm{mL}$ and $80.73 \mu \mathrm{g} / \mathrm{mL}$ respectively, $72 \mathrm{hrs}$ post exposure (Samwel et al., 2019). Furthermore, another study on Kotschya africana afforded the isolation of a triterpenoid, lupeol (Gitu, 2009). Lupeol is also known to be active against the larvae of Aedes aegypti with $\mathrm{LC}_{50}$ of $158.71 \mu \mathrm{g} / \mathrm{mL}$ after $24 \mathrm{hrs}$ exposure time (Nobsathian et al., 2018). Moreover, phytochemical profiling of crude ethanolic extract from leaves and roots of Kotschya strigosa, Kotschya speciosa and Kotschya thymodora identified the presence of a weak larvicidal compound, cycloartenone (Innocent et al., 2015). Literature review reveals no information about isolation of any phenolic acids from plant species belonging to the genus Kotschya. Nevertheless, a number of naturally occurring plant phenolic acids are known to be potent larvicides (Kishore et al., 2011, Pavela, 2011, Lomonaco et al., 2009). Furthermore, studies have shown that the larvicidal potential of phenolic acids is influenced by the presence of a hydrocarbon chain on the phenolic ring and its degree of unsaturation (Lomonaco et al., 2009). For instance, Carvalho et al., 2019, reported that at $100 \mu \mathrm{g} / \mathrm{mL}$ of salicylic acid possess mild larvicidal activity against Ae.egypti and $C x$. quinquefasciatus with cumulative mortality of $39 \%$ and $48 \%$ respectively $24 \mathrm{hrs}$ post exposure. In the same study salicylic acid bearing $O$ pentadecyl moiety (anacardic acid) showed to possess significantly higher larvicidal activity against $A e$. aegypti and $C x$. quinquefasciatus with $\mathrm{LC}_{50}$ of 5.93 $\mu \mathrm{g} / \mathrm{mL} 24 \mathrm{hrs}$ post exposure. In the same way, vanillic acid (1) is known to exhibit a weak larvicidal activity against the larvae of $C x$. quinquefasciatus with the 
$\mathrm{LC}_{50}$ above $300 \mu \mathrm{g} / \mathrm{mL}$ after $24 \mathrm{hrs}$ exposure time (Pavela, 2011) while protocatechuic acid (2) had cumulative mortality of $40 \%$ at $1.25 \mu \mathrm{g} / \mathrm{mg}$ at $24 \mathrm{hrs}$ exposure time, against adults females of Ae.egypti (Amin et al., 2012). The present study indicates that both the vanillic acid (1) and protocatechuic acid (2) possess a moderate mosquito larvicidal activity against An. gambiae s.s. (Table 2). In comparison to the larvicidal activity reported in the literature (Pavela, 2011,) both phenolic acids demonstrate higher larvicidal potential against $C x$. quinquefasciatus than for An. gambiae. The $\mathrm{LC}_{50}$ and $\mathrm{C}_{90}$ values of the two phenolic acids presented in table 2 show not statistically significant difference at $p \leq 0.05$ and this can be explained by the structural similarity amongst the two compounds. The two phenolic acids therefore have many physicochemical properties in common which can also reflect their biological effects (Kamel and Syam, 2013). In our previous work we reported the larvicidal potential of crude ethanolic extract from the leaves and roots $K$. thymodora against $C x$. quinquefasciatus and An. gambiae larvae. At 500 $\mu \mathrm{g} / \mathrm{mL}$, the crude ethanolic extracts of stems and roots from $K$. thymodora showed to cause cumulative mortality up to $70 \%$ of $C x$. quinquefasciatus larvae after 8 days of exposure (Innocent et al., 2012). The crude ethanolic extract from the leaves and roots of $K$. thymodora possesses activity against An. gambiae larvae with $\mathrm{LC}_{50}$ of $16.35 \mu \mathrm{g} / \mathrm{mL}$ and $53.35 \mu \mathrm{g} / \mathrm{mL}$ respectively after $48 \mathrm{hrs}$ exposure (Daniel et al., 2020). Comparatively the crude extract from the leaves exhibits higher larvicidal activity against An. gambiae s.s than vanillic acid (1) and protocatechuic acid (2). However, the presence of the two mild active larvicidal phenolic acids in the ethanolic extract of Kotschya thymodora leaves, together with the previously reported cycloartenone (Innocent et al., 2015), partly contributes to explaining the larvicidal potential of this plant.

Conclusion: This is the first report on the isolation and mosquito larvicidal studies against An. gambiae s.s of the two phenolic acids viz. vanillic acid (1) and protocatechuic acid (2) from plants belonging to the genus Kotschya. The larvicidal activity of these phenolic acids exhibit mild activity compared to the activity of leaf crude extracts of $K$. thymodora previously reported. Hence a call for further isolation of bioactive compounds from this plant species.

Competing interest: Authors of this work declare no competing interest.

Acknowledgement: This work was partly funded by DAAD In-Country/In-region scholarship program Tanzania, 2016 and the Research Council of Norway through the (226244/F50). Authors are also thankful to Institute of Traditional Medicines at MUHAS and the University of Bergen (UiB) for providing laboratories for conducting experiments.

\section{REFERENCES}

Amin, E; Radwan, MM; El-Hawary, SS; Fathy, MM; Mohammed, R; Becnel, JJ; Khan, I (2012). Potent insecticidal secondary metabolites from the medicinal plant Acanthus montanus. Rec. Nat. Prod. 6(3): 301-305.

An, LJ; Guan, S; Shi, GF; Bao, YM; Duan, YL; Jiang, B (2006). Protocatechuic acid from Alpinia oxyphylla against MPP+-induced neurotoxicity in PC12 cells. Food Chem. Toxicol. 44(3): 436-443.

Carvalho, FHG; Andrade, AM; Araújo, NC; Santos, LM; Castro, AN; Charneau S; Monnerat R; Santana, MJ; Bastos DMI (2019). Larvicidal and pupicidal activities of eco-friendly phenolic lipid products from Anacardium occidentale nutshell against arbovirus vectors. Environ. Sci. Pollut. Res. 26(6): 5514-5523.

Cox, FE (2010). History of the discovery of the malaria parasites and their vectors. Parasites Vectors. 3(1): 1-9.

Daniel, I; Innocent, E; Sempombe, J; Mugoyela, V; Samwel, B (2020). Mosquito larvicidal activity of polar extracts from three Kotschya species against Anopheles gambiae s.s. Int. J. Mosq. Res. 7(3): 29-33.

Ghareib, HRA; Abdelhamed, MS; Ibrahim, OH (2010). Antioxidative effects of the acetone fraction and vanillic acid from Chenopodium murale on tomato plants. Weed Biol. Manag. 10(1): 64-72.

Ghosh, A; Chowdhury, N; Chandra, G (2012). Plant extracts as potential mosquito larvicides. Indian $J$. Med. Res. 135(5): 581-598.

Gitu, LM (2009). Biological and phytochemical studies of medicinal plants, Antidesma venosum (Euphorbiaceae) and Kotschya africana (Fabaceae) used in traditional medicine in Kenya. $\mathrm{PhD}$ thesis. Department of Chemistry. Jomo Kenyatta University of Agriculture and Technology. Nairobi, Kenya.

Innocent, E; Kaale, E; Mbwambo, Z (2012). Larvicidal activities of five Kotschya species 
against Culex quinquefasciatus Say (Culicidae: Diptera). Int. J. Biol. Chem. 6(2): 603-612.

Innocent, E; Sempombe, J; Masimba, JP; Daniel, I; Heydenreich, M (2015). Comparison of cycloartenone from four insecticidal Kotschya species (Fabaceae) harvested during dry and wet seasons. Int. J. Pharmacogn. Phytochem. Res. 4(4): 97-102.

Kamel, MM; Syam, MY (2013). Structure and physicochemical properties in relation to drug action. Egypt. Pharm. J. (12): 95-108.

Kimbi, HK (2012). Environmental factors and preventive methods against malaria parasite prevalence in rural Bomaka and Urban Molyko, South-west Cameroon. Int. J. Parasitol. 04(01): $1-5$.

Kishore, N; Mishra, BB; Tiwari, KV; Tripathi, V (2011). A review on natural products with mosquitocidal potentials. Opportunity, challenge and scope of natural products in medicinal chemistry, p 335-365.

Kokwaro, G (2009). Ongoing challenges in the management of malaria. Malar. J. 8(Suppl 1):S2.

Léong, MJ; Duchemin, BJ; Marrama, L; Rabarison, P; Goff, LG; Rajaonarivelo V; Robert V (2003). Distribution of the species of the Anopheles gambiae complex and first evidence of Anopheles merus as a malaria vector in Madagascar. Malar. J. 2(33): 1-7.

Lomonaco, D; Santiago, PMG; Ferreira, SY; Arriaga, CMA; Mazzetto, ES; Mele, G; Vasapollo, G (2009). Study of technical CNSL and its main components as new green larvicides. Green Chem. 11(1): 31-33.
Nobsathian, S; Bullangpoti, V; Kumrungsee, N; Wongsa, N; Ruttanakum, D (2018). Larvicidal effect of compounds isolated from Maerua siamensis (Capparidaceae) against Aedes aegypti (Diptera: Culicidae) larvae. Chem. Biol. Technol. Agric. 5(1): 4-10.

Pavela, R (2011). Insecticidal properties of phenols on Culex quinquefasciatus Say and Musca domestica L. Parasitol. Res. 109(6): 1547-1553.

Pavela, R (2016). History, presence and perspective of using plant extracts as commercial botanical insecticides and farm products for protection against insects. Int. J. Plant Prot. 52(4): 229-241.

Samwel, B; Innocent, E; Machumi, F; Kisinza, NW; Heydenreich, M (2019). Isolation and characterization of mosquito larvicidal compounds from leaves of Kotschya uguenensis (Taub). Int. J. Phytomedicine. 7(6): 1-4.

Teklehaimanot, A; Mejia, P (2008). Malaria and poverty. Ann. N. Y. Acad. Sci. 1136: 32-37.

World Health Organization (2019). World malaria report. WHO library cataloguing in publication (CIP) data. p 4-11.

Yewhalaw, D; Wassie, F; Steurbaut, W; Spanoghe, P; Bortel, VW; Denis, L; Tessema, AD; Getachew, Y; Coosemans, M; Duchateau, L; Speybroeck, N (2011). Multiple insecticide resistance: An impediment to insecticide-based malaria vector control program. PLoS One. 6(1): 16066. 